\title{
Interaction of N-(2-Methyl Thiophenyl)-2-Hydroxy-1- Naphthaldimine with Tin Dioxide Nanoparticles: A Spectroscopic Approach
}

\author{
Suvetha Rani Jayaprakash, Ramakrishnan Veerabahu \\ Department of Laser Studies, School of Physics, Madurai Kamaraj University, Madurai, India \\ Email: nsuvetha@yahoo.co.in
}

Received May 16, 2012; revised June 17, 2012; accepted June 28, 2012

\begin{abstract}
The interaction of $\mathbf{N}$-(2-methyl thiophenyl)-2-hydroxy-1-naphthaldimine (NMTHN) with tin dioxide nanoparticles $\left(\mathrm{SnO}_{2}\right.$ NPs) has been investigated by spectroscopic tools such as absorption and fluorescence spectroscopy. Absorption spectroscopy reveals the formation of ground state complex. Fluorescence spectroscopy has been used to study the signatures of fluorescence quenching. $\mathrm{SnO}_{2} \mathrm{NPs}$ are found to quench the intrinsic fluorescence of NMTHN via static and dynamic quenching. The deviation from linearity in the Stern-Volmer plot has been observed.
\end{abstract}

Keywords: N-(2-Methyl Thiophenyl)-2-Hydroxy-1-Naphthaldimine; Tindioxide Nanoparticles; Optical Absorption; Fluorescence Quenching

\section{Introduction}

Owing to their potential application in solar energy conversion, semiconductor nanoparticles have been extensively studied from both experimental and theoretical viewpoints [1-4]. Variety of techniques including spectroscopy, microscopy and X-ray techniques has been used to characterize the properties of nanoparticles. Most of the studies have been focused on their equilibrium properties such as absorption and emission, particle shape, surface structure, inter particle interaction, self assembly, and formation of superlattices [5]. Tindioxide $\left(\mathrm{SnO}_{2}\right)$ is a n-type metal oxide semiconductor with the wide bandgap of $3.6 \mathrm{eV}$. Because of its remarkable electrical, optical and electrochemical properties, $\mathrm{SnO}_{2}$ serves a wide range of applications in solar cells, catalytic supporting materials, transparent electrodes and solid state chemistry [6]. The higher electron mobility $(\sim 100-$ $200 \mathrm{~cm}^{2} \cdot \mathrm{v}^{-1} \cdot \mathrm{s}^{-1}$ ) of $\mathrm{SnO}_{2}$ NPs proposes a faster diffusion of photo induced electrons in $\mathrm{SnO}_{2}$ and its larger band gap would be creating fewer oxidation holes in the valence band. Thus low sensitivity of $\mathrm{SnO}_{2}$ to UV degradation facilitates the long-term stability of dye senstitized solar cells. The low isoelectric point (at $\mathrm{pH} 4-5$ ) of $\mathrm{SnO}_{2}$ leads to less absorption of the dye with acidic carboxyl groups $[7,8]$. The ability of organic dyes to sensitize large band gap semiconductor materials has been used for the design of light energy conversion devices [9]. Schiff bases play an important part in the development of co-ordination chemistry. The common structural feature of these compounds is the azomethine group with a general formula $\mathrm{RHC}=\mathrm{N}-\mathrm{R}$ ', where $\mathrm{R}$ and $\mathrm{R}$ ' are alkyl, aryl, cyclo alkyl or heterocyclic group which may be variously substituted. They are easily prepared in general by the condensation reaction of primary amines with carbonyl compounds [10]. In the field of co-ordination chemistry, Schiff bases from 2-hydroxy 1-napthaldehyde have been used as chelating ligands. $\mathrm{N}-\mathrm{H} \cdots \mathrm{O}$ (keto form) and $\mathrm{N} \cdots \mathrm{H}-\mathrm{O}$ (enol form) are the two types of intra molecular hydrogen bonds in Schiff bases. Both types of hydrogen bonds were found in the aldimine compounds derived from 2-hydroxy 1-Naphthaldehyde [11]. The existence of tautomerism between these two types of bonds created a great interest in 2-hydorxy Schiff base ligands. The ortho hydroxyl naphthalidene anilines show two bands above $400 \mathrm{~nm}$ in the visible region which are assigned to the keto form. In naphthaldimines both forms keto/enolimino are possible and $\mathrm{O}-\mathrm{H} \cdots \mathrm{N}$ or $\mathrm{N}-\mathrm{H} \cdots \mathrm{O}$ intra molecular hydrogen bonds can occur [12]. If combined with chelating activities, Schiff base may become a promising dye sensitizer in molecular photovoltaic cells [13].

Semiconducting oxides such as $\mathrm{TiO}_{2}$ and $\mathrm{SnO}_{2}$ directly interact with the excited dye molecules thus inducing heterogeneous electron transfer at the semiconductor/dye interface. This interesting property of sensitizing dyes is useful in the design of photochemical solar cells.

A process which decreases the fluorescence intensity of a given substance is known as quenching. It may also 
result from a photo-induced electron transfer process between the excited dye and the nanoparticles. Our group has studied spectral investigation of NMTHN by silver nanoparticle using fluorescence quenching [14]. Although there are many studies on the photochemical and fluorescence behavior of organic dyes on $\mathrm{SnO}_{2}$ thin film, there is none for $\mathrm{SnO}_{2} \mathrm{NPs}$ on the fluorescence quenching of Schiff base in methanol medium [15-18]. In the present study, using optical absorption and fluorescence emission techniques the effect of $\mathrm{SnO}_{2}$ NPs on NMTHN has been investigated.

\section{Experimental}

\subsection{Materials}

All chemicals that are used in this work were obtained from Merck with $99.9 \%$ purity and also used without further purification. The procedure of synthesis of NMT$\mathrm{HN}$ (Figure 1) is described as follows [14].

2-hydroxy 1-napthldehyde (1.72 g, $10 \mathrm{mmol})$ was dissolved in alcohol and treated with an alcoholic solution of 2-(methylthio) aniline $(1.39 \mathrm{~g}, 10 \mathrm{mmol})$. The content of the above solution was kept at room temperature over night. The formation of Schiff base took place slowly with good yield. The pure brownish yellow crystals of the Schiff base was filtered, washed with alcohol and dried.

$\mathrm{SnO}_{2}$ nanoparticles used in this study were synthesized as follows: Solution of $\mathrm{SnCl}_{2} \cdot 5 \mathrm{H}_{2} \mathrm{O}$ (4.5126 g) of $0.1 \mathrm{M}$ was prepared in the de-ionized water $(200 \mathrm{ml})$ to get a mixed aqueous solution. To this mixed aqueous solution, ammonia solution was added into drop wise under vigorous stirring to get the $\mathrm{pH}$ value of the solution in the range of $8-9$. Now, the precipitate had been formed at the bottom of the glass beaker. For about 2 hours the precipitate was kept at room temperature for ageing and then washed with deionized water. The washing was repeated for a number of $5-6$ times. The resulting precipitate was heated at $80^{\circ} \mathrm{C}$ for about 5 hours. The dried precipitate was kept at $105^{\circ} \mathrm{C}$ for 4 hours, and it was loaded into the alumina crucible. Then for about

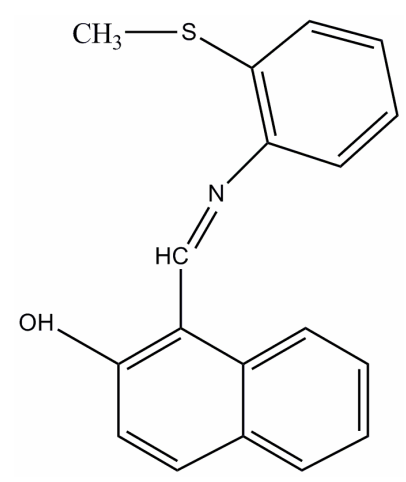

Figure 1. Molecular structure of N-(2-methyl thiophenyl)-2hydroxy-1-napthaldimine (NMTHN).
5 hours in air it was annealed in a muffle furnace at $600^{\circ} \mathrm{C}$ to enhance the crystallinity of $\mathrm{SnO}_{2} \mathrm{NPs}$ and the product appeared as white in color after the heat treatment. The particle size of the resultant product was found as $160 \mathrm{~nm}$ using micro Raman spectroscopic technique [19].

\subsection{Apparatus}

At room temperature using $1 \mathrm{~cm}$ path length rectangular quartz cell by means of UV-Vis absorption spectrophotometer (Shimadzu UV 2450) and Spectrofluorophotometer (Shimadzu RC 5301-PC), steady state optical absorption and fluorescence emission spectra of the samples were recorded. While the concentration of $\mathrm{SnO}_{2} \mathrm{NPs}$ ranged from 0.5 to $0.9 \mathrm{mM}$, the concentration of NMTHN in methanol was $0.01 \mathrm{mM}$ throughout the experiment and was precisely maintained the same in all samples. The optical absorption and fluorescent measurements have been repeated for five times for each set of samples. It was noticed that the data are reproducible with an accuracy of $\pm 0.1 \mathrm{~nm}$. And hence, there is a good reliability of the data.

\section{Results}

\subsection{Absorption Spectroscopy}

Figure 2 shows the UV-Vis absorption spectrum of NMTHN in methanol. The absorption maximum occurs at $441 \mathrm{~nm}$. The entire spectrum undergoes a hyper chromic effect with a little spectral shift (Figure 3), with each addition of $\mathrm{SnO}_{2} \mathrm{NPs}$ concentration.

\subsection{Fluorescence Spectroscopy}

To determine the nature of interaction between NMTHN and $\mathrm{SnO}_{2} \mathrm{NPs}$ fluorescence spectroscopy is used. Figure 4 shows the steady state fluorescence emission spectrum

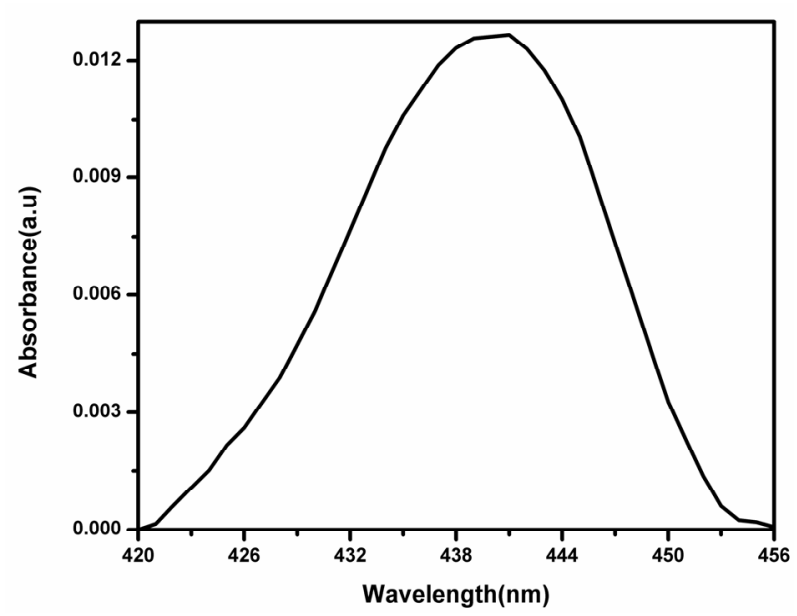

Figure 2. Absorbance spectra of NMTHN in methanol. 


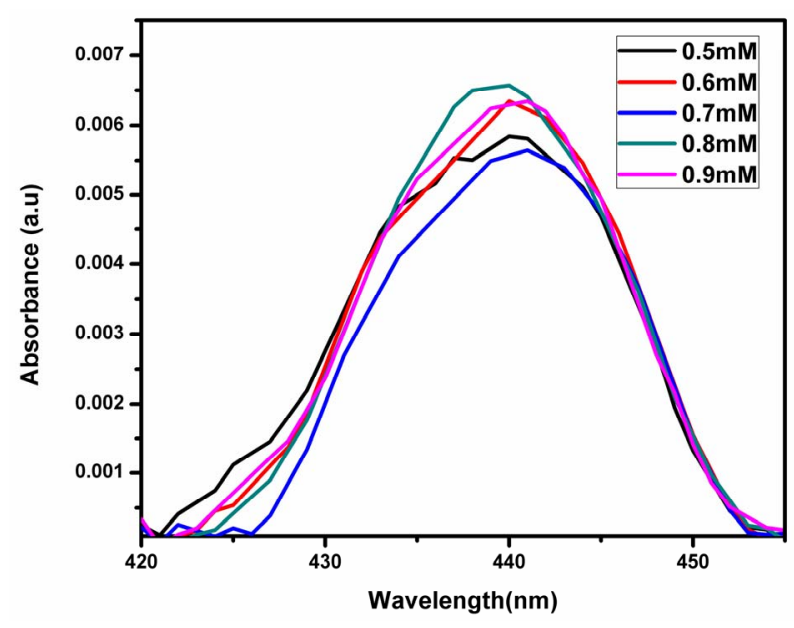

Figure 3. Absorbance spectra of NMTHN with various concentration of $\mathrm{SnO}_{2} \mathrm{NPs}$ in methanol.

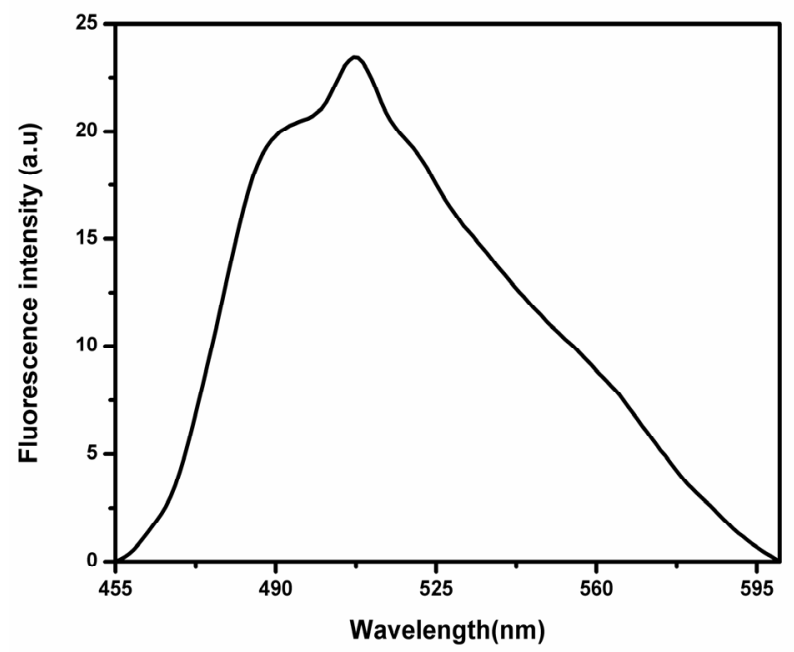

Figure 4. Fluorescence spectra of NMTHN in methanol.

of the NMTHN in methanol. The emission maxima occur at $507 \mathrm{~nm}$ and also it is observed that the intensity of fluorescence emission band of Schiff base is found to change with the concentration of $\mathrm{SnO}_{2} \mathrm{NPs}$ (Figure 5).

\section{Discussion}

The absorption spectra in Figure 3 show a minor blue shift with the addition of $\mathrm{SnO}_{2} \mathrm{NPs}$ in methanol from the absorption maximum of pure Schiff base in methanol at $441 \mathrm{~nm}$. This shift may be ascribed to the concentration dependence of the fluorophore. The observed concentration dependence of the flurophore may at least partially result from the change in the ground state of the NMTHN adsorbed on the NP surface [20]. The observed decrease in fluorescence intensity in Figure 5 may be due to one of the following reasons: 1) quenching mechanisms (static/dynamic); 2) scattering loss; 3) inner filter effect. The occurrence of fluorescence quenching

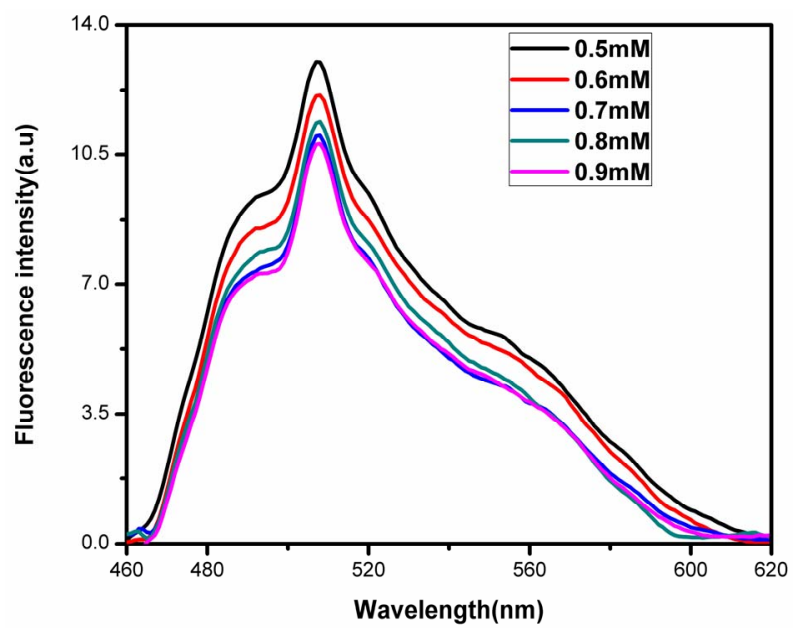

Figure 5. Fluorescence spectra of NMTHN with various concentration of $\mathrm{SnO}_{2}$ NPs in methanol.

depends upon the mechanism, which in turn depends upon the structure of individual molecules. Detailed analysis of the mechanism of quenching is complex. Commonly, the static and dynamic (collisional) quenching mechanisms are the two mechanisms used to analyze the fluorescence quenching processes. In dynamic quenching, the ratio of intensities is linearly related to the quencher concentration as defined by the Stern-Volmer equation

$$
\frac{F_{0}}{F}=1+K_{s v}[Q]
$$

where $F_{0}$ and $F$ are the fluorescence intensities of Schiff base in absence and presence of quencher concentration $[Q]$ respectively. In dynamic process, energy can be transferred nonradiatively and this collisional mechanism assumes the electron transfer from excited state fluorophores. In the static quenching process, the fluorophore and quencher molecules are in the ground state. The fluorophore and quencher molecules bind together to form a ground state complex and a ground state equilibrium between the fluorophore and quencher is assumed or perfect quenching within the sphere of action [21]. To evaluate the Stern-Volmer quenching constant $K_{S V}$, the range within which the Stern-Volmer plot (Figure 6) is linear is considered and $K_{S V}$ is found out to be $1.6 \times 10^{3}$ $\mathrm{M}^{-1}$. The observed $10 \%$ error suggests that the signature is different. The Stern-Volmer plot (Figure 6) shows the positive deviation from straight line.

Generally the positive deviation from linearity of Stern-Volmer plot occurs when 1) combination of static and dynamic quenching takes place and/or 2) degree of quenching is large [22]. By carefully analyzing the absorption spectra of the fluorophore, one can distinguish the static and dynamic quenching. Collisional quenching only affects the excited state of the fluorophore and thus no changes in the absorption spectra are predicted. How- 


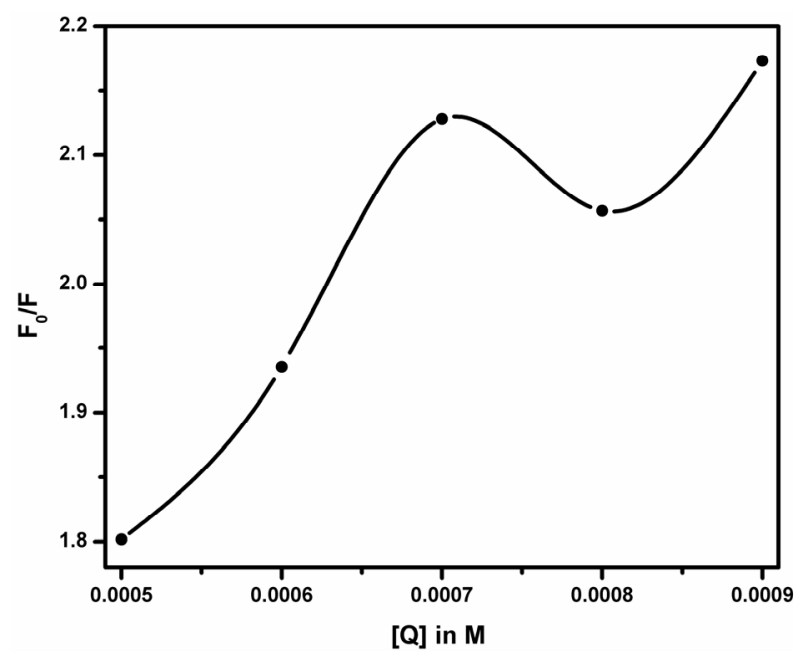

Figure 6. Stern-Volmer plot between the concentration of the quencher $(Q)$ and the fluorescence intensity ratio $\left(F_{0} / F\right)$.

ever the ground state complex formation results in perturbation of the absorption spectrum. It is observed that there is no formation of new band in Figure 5 but a decrease in the fluorescence intensity. Thus there is no evidence for the formation of the exciplex. In the absence of any chemical reaction of the excited fluorophore and the quencher, the positive deviation from the SternVolmer relation may also be due to ground state complex formation. Figure 6 gives the static constant value as approximately equal to $1.6 \times 10^{3} \mathrm{M}^{-1}$. Therefore, $\mathrm{SnO}_{2}$ NPs concentration of nearly $0.6 \mathrm{mM}$ is necessary to complex $50 \%$ of the fluorophore. The static component is not only due to complexation but $\mathrm{SnO}_{2} \mathrm{NP}$ being near to the fluorophore at the fraction of moment of excitation. The percentage of such complexed fluorophore increases as the mole fraction of $\mathrm{SnO}_{2}$ in the solution increases. Thus the sphere of action static quenching model may be employed for data analysis in heterogeneous systems.

The only observable fluorophores $(1-W)$ are those for which there are no adjacent quenchers. The modified form of the Stern-Volmer equation

$$
\frac{\left[1-\frac{F}{F_{0}}\right]}{[Q]}=K_{s v}\left(\frac{F}{F_{0}}\right)+\frac{(1-W)}{[Q]}\left(1-\left(F / F_{0}\right)\right)\left(1-\left(F / F_{0}\right)\right)
$$

is used to calculate the fraction of fluorophores which do not contain a quencher within their surrounding sphere of action. The factor $W$ in the modified Stern-Volmer equation is approximately equal to $\exp (-V[Q])$, where $V$ is the static quenching constant and it represents active volume element $\left(V / N^{\prime}\right)$ surrounding the excited fluorophore. When a quencher turns out to reside within a sphere of action with a volume of $V / N^{\prime}$, where $N^{\prime}$ is the Avagadro's number and $r$ (kinetic distance), surrounding a fluorophore at the time of excitation, instantaneous quen- ching occurs. On excitation of the fluorophore, a quencher particle which is already within this volume will be able to quench the fluorescence without the requirement of collisional interaction. The probability of the quencher being within this volume at the time of excitation depends on the volume $V$ and on the quencher concentration [23]. The modified Stern-Volmer plot $\frac{\left[1-\frac{F}{F_{0}}\right]}{[Q]}$ against $F / F_{0}$ for steady state is drawn and also found to be perfectly not a linear one as shown in Figure 7. Since the Figures $\mathbf{6}$ and $\mathbf{7}$ are made out of the data of fluorescence measurements, we could observe the same trend with reproducibility.

The Stern-Volmer quenching constant was obtained as $K_{S V}=2.6 \times 10^{3} \mathrm{M}^{-1}$ with an error of $35 \%$ using least square fit method by determining the slope. Equation $W$ $=\exp (-V[Q])$ is employed to deduce the intercept, the value of $W$ and hence the static quenching constant $V=$ $412.2 \mathrm{M}^{-1}$ with $1 \%$ error and in turn the kinetic distance i.e., the radius of the sphere of action is determined as $5.47 \pm 0.1 \mathrm{~nm}$ from the plot (Figure 8) between $\ln W$ and the quencher concentration $[Q]$. It is observed that the value of $K_{S V}$ is large compared with $V$, which explains the lack of major absorption change on addition of the quencher [24]. The radii of the quencher as determined by the Micro-Raman spectroscopic technique [19] is equal to $80 \mathrm{~nm}$ which is greater than that of the kinetic distance. This verifies that the quenching is not due to the sphere of action. The experimental scattering cross-section of $\mathrm{SnO}_{2} \mathrm{NPs}$ in methanol medium is calculated using the formula

$$
\sigma=\frac{(\ln 10) D}{\rho L}
$$

where $D$ is the optical density and $\rho$ is the number density and $L$ is the optical path length [25] and it has the order of $10^{-23} \mathrm{~m}^{2}$. Though the scattering cross section area is very small, the higher concentration of NPs $(0.05$ $\mathrm{mM}-0.9 \mathrm{mM})$ than that of Schiff base $(0.01 \mathrm{mM})$ may also contribute in the form of inner filter effect. In that case, the intensity of the excitation light is not constant throughout the solution. As a result, the intensity of emitted light could also change. Thus we conclude that the positive deviation of the Stern-Volmer plot suggests the existence of both static and dynamic quenching that cannot be distinguished as the system is heterogeneous. In the case of NMTHN-Silver nanoparticle, only one type of quenching has been observed. Surface plasmon effect will be a predominate one in the case of silver nanoparticels. As the possibility of formation of static quenching complex has been ruled out, the dominance of collisional quenching is due to the small particle size 


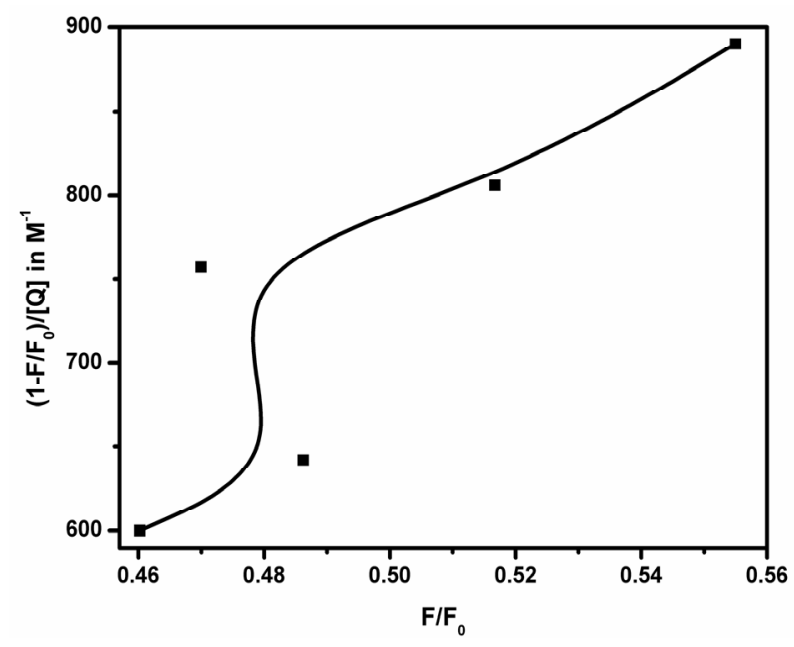

Figure 7. Modified Stern-Volmer $\operatorname{plot}\left(1-\left(F / F_{0}\right)\right) /[Q]$ against $\mathbf{F} / \mathbf{F}_{0}$.

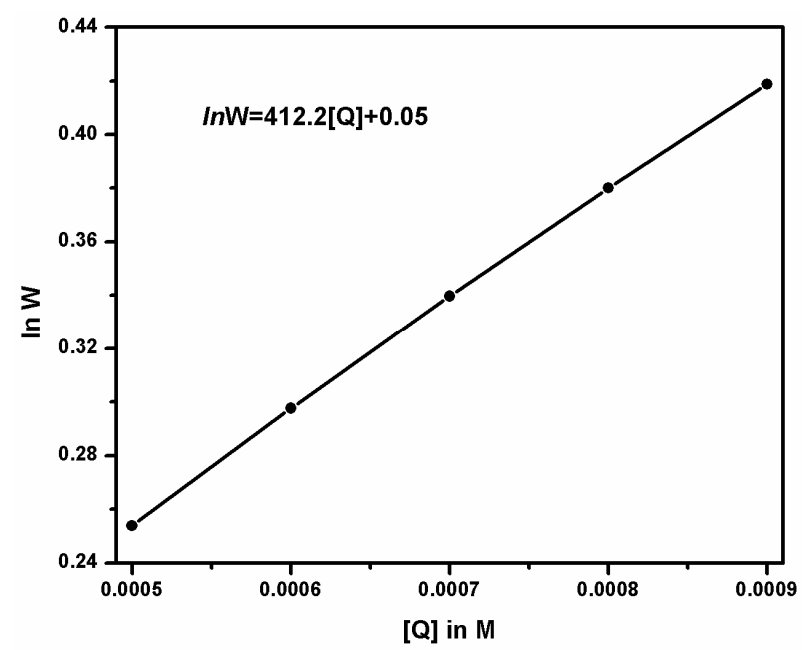

Figure 8. Plot of $\ln W$ against $[Q]$.

$(<50 \mathrm{~nm})$ [14]. In the present case, the $\mathrm{SnO}_{2}$ particle size is greater than $150 \mathrm{~nm}$. So, this could also be a reason that the linearity of the Stern-Volmer plot is not observed.

\section{Conclusions}

From the present investigation we conclude that: 1) the formation of the ground state complex between NMTHN and $\mathrm{SnO}_{2}$ NPs and 2) the observed deviation in SternVolmer plot is due to the simultaneous presence of static and dynamic quenching components and also by the presence of higher concentration of the quenchers.

\section{Acknowledgements}

The authors thank CSIR, Government of India for the financial assistance in the form of a research scheme to one of the authors (VRK). Further the financial support received from UGC, Government of India under DRS
Phase III to have Shimadzu UV-2450 UV-visible spectrophotometer facility is gratefully acknowledged. J. S would like to express her sincere thanks to the Principal and Management of the Lady Doak College for their encouragement and Ms P. Sangeetha, Department of Laser Studies, Madurai Kamaraj University for the help on the preparation of $\mathrm{SnO}_{2}$ nanoparticles.

\section{REFERENCES}

[1] R. S. Ningthoujam and S. K. Kulshreshtha, "Nanocrystalline $\mathrm{SnO}_{2}$ from Thermal Decomposition of Tin Citrate Crystal: Luminescence and Raman Studies," Materials Research Bulletin, Vol. 44, No. 1, 2009, pp. 57-62. doi:10.1016/j.materresbull.2008.04.004

[2] S. G. Ansari, P. Boroojerdian, S. R. Sainker, R. N. Karekar, R. C. Aiyer and S. K. Kulkarni, "Grain Size Effects on $\mathrm{H}_{2}$ Gas Sensitivity of Thick Film Resistor Using $\mathrm{SnO}_{2}$ Nanoparticles," Thin Solid Films, Vol. 295, No. 1-2, 1997, pp. 271-276. doi:10.1016/S0040-6090(96)09152-3

[3] X. Peng, M. C. Schlamp, A. V. Kadavanich and A. P. Alivisatos, "Epitaxial Growth of Highly Luminescent $\mathrm{CdSe} / \mathrm{CdS}$ Core/Shell Nanocrystals with Photostability and Electron Accessibility," Journal of the American Chemical Society, Vol. 119, No. 30, 1997, pp. 7019-7029. doi:10.1021/ja970754m

[4] W.-Y. Chung, D.-D. Lee and B.-K. Sohn, "Effects of Added $\mathrm{TiO}_{2}$ on the Characteristics of $\mathrm{SnO}_{2}$ Based Thick Film Gas Sensors," Thin Solid Films, Vol. 221, No. 1-2, 1992, pp. 304-310. doi:10.1016/0040-6090(92)90832-V

[5] J. Z. Zhang, "Interfacial Charge Carrier Dynamics of Colloidal Semiconductor Nanoparticles," Journal of Physical Chemistry B, Vol. 104, No. 31, 2000, pp. 72397253. doi:10.1021/jp000594s

[6] S. Ferrere, A. Zaban, and B. A. Gregg, "Dye Sensitization of Nanocrystalline Tin Oxide by Perylene Derivatives," Journal of Physical Chemistry B, Vol. 101, No. 23, 1997, pp. 4490-4493._doi:10.1021/jp970683d

[7] J. F. Qian, P. Liu, Y. Xiao, Y. Jiang, Y. L. Cao, X. P. Ai and H. X. Yang, "TiO 2 Coated Multilayered $\mathrm{SnO}_{2}$ Hollow Microspheres for Dye-Sensitized Solar Cells," Advanced Materials, Vol. 21, No. 36, 2009, pp. 3663-3667.

[8] S. Gubbala, V. Chakrapani, V. Kumar and M. K. Sunkara, "Band Edge Engineered Hybrid Structures for Dye-Sensitized Solar Cells Based on $\mathrm{SnO}_{2}$ Nanowires," Advanced Functional Materials, Vol. 18, No. 16, 2008, pp. 24112418. doi:10.1002/adfm.200800099

[9] S. Das and P. V. Kamat, "Can H-Aggregates Serve as Light Harvesting Antennae? Triplet-Triplet Energy Transfer between Excited Aggregates and Monomer Thionine in Aerosol-OT Solutions," Journal of Physical Chemistry $B$, Vol. 103, No. 1, 1999, pp. 209-215. doi:10.1021/jp983816j

[10] A. Blagus, D. Cincic, T. Friscic, B. Kaitner and V. Stilinovic, "Schiff Bases Derived from Hydroxyaryl Aldehydes: Molecular and Crystal Structure, Tautomerism, Quinoid Effect, Coordination Compounds," Macedonian Journal of Chemistry and Chemical Engineering, Vol. 29, 
No. 2, 2010, pp. 117-138.

[11] H. Unver and T. Nuri Durlu, "Crystal Structure and Conformational Analysis of 1-[N-2-Bromophenyl Naphthaldimine," Journal of Molecular Structure, Vol. 655, No. 3, 2003, pp. 369-374. doi:10.1016/S0022-2860(03)00277-1

[12] A. M. Asiri and K. O. Badahdah, "Synthesis of Some New Anils: Part 1. Reaction of 2-Hydroxy-Benzaldehyde and 2-Hydroxy Naphthaldehyde with 2-Aminopyridene and 2-Aminopyrazine," Molecules, Vol. 12, 2007, pp. 1796-1804.

[13] H. Dincalp, S. Yavuz, O. Hakli, C. Zafer, C. Ozsoy, I. Durucasu and SiddikIcli, "Optical and Photovoltaic Properties of Salicylaldimine Based Azo Ligands," Journal of photochemistry and Photobiology A: Chemistry, Vol. 210, No. 1, 2010, pp. 8-16.

doi:10.1016/j.jphotochem.2009.12.012

[14] P. Manikandan and V. Ramakrishnan, "Spectral Investigations on N-(2-Methyl Thiophenyl) 2-Hydroxy-1-Naphthaldimine by Silver Nanoparticles: Quenching," Journal of Fluorescence, Vol. 21, No. 2, 2011, pp. 693-699. doi:10.1007/s10895-010-0757-3

[15] I. Bedja, S. Hotchandani and P. V. Kamat, "Preparation and Photochemical Characterization of Thin $\mathrm{SnO}_{2} \mathrm{Na}-$ nocrystalline Semiconductor Films and Their Sensitization with Bis(2,2'-bipyridine) (2,2'-bipyridine-4,4'-dicarboxylic acid) Ruthenium (II) Complex," Journal of Physical Chemistry, Vol. 98, 1994, pp. 4133-4140. doi:10.1021/j100066a037

[16] H. J. Snaith and C. Ducati, " $\mathrm{SnO}_{2}$ Based Dye Sensitized Hybrid Solar Cells Exhibiting near Unity Absorbed Photon to Electron Conversion Efficiency," Nanoletters, Vol. 10, No. 4, 2010, pp. 1259-1265. doi:10.1021/n1903809r

[17] D. Liu, R. W. Fessenden, G. L. Hug and P. V. Kamat, "Dye Capped Semiconductor Nanoclusters. Role of Back Electron Transfer in the Photosensitization of $\mathrm{SnO}_{2}$ Nanocrystallites with Cresyl Violet Aggregates," Journal of Physical Chemistry B, Vol. 101, No. 14, 1997, pp. 2583-
2590. doi:10.1021/jp962695p

[18] C. Nasr, D. Liu, S. Hotchandani and P. V. Kamat, "Dye Capped Semiconductor Nanoclusters. Excited State and Photosensitization Aspects of Rhodamine 6G H-Aggregates Bound to $\mathrm{SiO}_{2}$ and $\mathrm{SnO}_{2}$ Colloids," Journal of Physical Chemistry, Vol. 100, No. 26, 1996, pp. 1105411061. doi:10.1021/jp9537724

[19] P. Sangeetha, V. Sasirekha and V. Ramakrishnan, "Micro Raman Investigation of Tin Dioxide Nanostructured Material Based on Annealing Effect," Journal of Raman Spectroscopy, Vol. 42, No. 8, 2011, pp. 1634-1639.

[20] S. Barazzouk, H. Lee, S. Hotchandani and P. V. Kamat, "Photosensitization Aspects of Pinacyanol H-Aggregates. Charge Injection from Singlet and Triplet Excited States into $\mathrm{SnO}_{2}$ Nanocrystallites," Journal of Physical Chemistry B, Vol. 104, No. 15, 2000, pp. 3616-3623. doi:10.1021/jp994311b

[21] I.-Y. S. Lee and H. Suzuki, "Quenching Dynamics Promoted by Silver Nanoparticles," Journal of Photochemistry and Photobiology A: Chemistry, Vol. 195, No. 2-3, 2008, pp. 254-260. doi:10.1016/j.jphotochem.2007.10.009

[22] B. Chakraborty and S. Basu, "Interaction of BSA with Proflavin: A Spectroscopic Approach," Journal of Luminescence, Vol. 129, No. 1, 2009, pp. 34-39. doi.10.1016/j.jlumin.2008.07.012

[23] J. R. Lakowicz, "Principles of Fluorescence Spectroscopy," 3rd Edition, Springer Science, New York, 2010.

[24] H. M. Suresh Kumar, R. S. Kunabenchi, J. S. Biradar, N. N. Math, J. S. Kadadevarmath and S. R. Inamdar, "Analysis of Fluorescence Quenching of New Indole Derivative by Aniline Using Stern-Volmer Plots," Journal of Luminescence, Vol. 116, No. 1-2, 2006, pp. 35-42. doi.10.1016/j.jlumin.2005.02.012

[25] A. J. Cox, A. J. DeWeerd and J. Linden, "An Experiment to Measure Mie and Rayleigh Total Scattering cross Sections," American Journal of Physics, Vol. 70, No. 6, 2002, pp. 620-625. doi:10.1119/1.1466815 